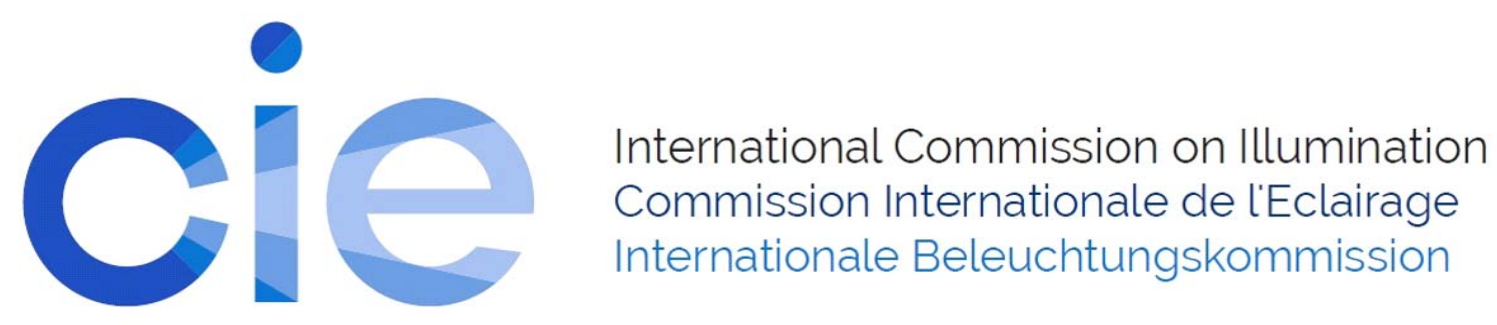

P0102

DOES A SINGLE LED BIN REALLY REPRESENT A SINGLE LED TYPE?

Thomas Merelle et al.

DOI 10.25039/x46.2019.PO102

from

CIE x046:2019

Proceedings

of the

29th CIE SESSION

Washington D.C., USA, June 14 - 22, 2019

(DOI 10.25039/x46.2019)

The paper has been presented at the 29th CIE Session, Washington D.C., USA, June 14-22, 2019. It has not been peer-reviewed by CIE.

(C) CIE 2019

All rights reserved. Unless otherwise specified, no part of this publication may be reproduced or utilized in any form or by any means, electronic or mechanical, including photocopying and microfilm, without permission in writing from CIE Central Bureau at the address below. Any mention of organizations or products does not imply endorsement by the CIE.

This paper is made available open access for individual use. However, in all other cases all rights are reserved unless explicit permission is sought from and given by the CIE.

CIE Central Bureau

Babenbergerstrasse 9

A-1010 Vienna

Austria

Tel.: +4317143187

e-mail: ciecb@cie.co.at

www.cie.co.at 


\title{
DOES A SINGLE LED BIN REALLY REPRESENT A SINGLE LED TYPE?
}

\author{
Merelle, T. ${ }^{1}$, Sari, J.K. ${ }^{2}$, Di Bucchianico, A. ${ }^{2}$, Onushkin, G. ${ }^{3}$, Bornoff, R. ${ }^{4}$, Farkas, G., ${ }^{5}$, Gaál, L. ${ }^{5}$, \\ Hantos, G. ${ }^{6}$, Hegedüs, J. ${ }^{6}$, Poppe, A. ${ }^{5,6}$ \\ ${ }^{1}$ Pi Lighting, SWITZERLAND, ${ }^{2}$ TU Eindhoven, NETHERLANDS, ${ }^{3}$ Signify (ex-Philips Lighting), \\ NETHERLANDS, ${ }^{4}$ Mentor, a Siemens business (ex Mentor Graphics), UNITED KINGDOM, ${ }^{5}$ Mentor, \\ a Siemens business (ex Mentor Graphics), HUNGARY, ${ }^{6}$ Budapest University of Technology and \\ Economics, HUNGARY
}

poppe@eet.bme.hu

DOI 10.25039/x46.2019.PO102

\begin{abstract}
In a recent H2020 European wide research project (Delphi4LED, www.delphi4LED.eu) extensive study for generating multi-domain compact LED models based on measurement data was concluded. In this context combination of the latest LED testing standards and recommendations of JEDEC and CIE have been applied in testing and new models and modelling methods have been developed in order to capture the multi-domain operation of LEDs for the purpose of simulation. A valid question is: how representative a model is for a type of an LED package, how to select a nominal LED device for modelling and how to capture the inherent variation of LED operating parameters. We had specific tasks dedicated to measure and model these variations. Findings related to capturing, quantifying and modelling of the thermal features of the mechanical structure of LED packages have already been published. In this paper we report about the measured variances of the multi-domain chip level operation of LEDs and the way how to model them. During this analysis we were confronted with the issue of having various parameter distributions in a LED population purchased from the same bin. This raises the question: LEDs belonging to a given binning class really represents a single LED type if their multi-domain behaviour is different.
\end{abstract}

Keywords: LED characterization, variability of LED parameters, LED multi-domain model parameters

\section{Motivation, specific objective}

Design of today's LED based applications such LED lamps with tuneable spectral power distribution for human centric lighting or temperature compensation in constant light output control of the most energy-efficient streetlighting applications or the latest LED based gas discharge lamp replacement solutions require careful design supported by extensive computer simulation in all operating domains (electrical, thermal, optical) of LEDs, at all levels of integration, from chip up to luminaire and lighting system level. At each level appropriate models (in an Industry 4.0 approach considered as digital twins of the physical devices) are needed, with the highest possible level of accuracy. To address this, a complete testing, modelling and simulation workflow has been worked out and proposed and published [Marty et al 2018], [Martin et al 2019].

LEDs have an inherent large variability in every operating parameter. For example, forward voltage (and power dissipation) has a typical $\pm 10 \%$ variability between two LEDs of the same part number (when driven at constant current). The same applies to radiant flux, lumen equivalent (efficacy of radiation) or junction-to-pad thermal characterization parameter (in this case up to $50 \%$ variability). Physical placement of the chip within the package, with the associated tolerances, also impacts the light distribution, etc.

Therefore, an essential part of implementing a workflow for the design of LED luminaires with an industrial approach in mind is, how to consider of sample-to-sample variations of LED packages selected from a given (even rather narrow) binning class of an LED package type. In the subsequent sections we shall give a brief overview of modelling LED packages with a summary of model parameters, and then we shall provide aspects of selecting a "median 
device" from an LED population, followed by some results of the statistical analysis of the distributions of the model parameters.

\section{Multi-domain characterization and modelling of LED packages}

\subsection{Measurement of LED characteristics}

Multi-domain characterization of LED packages means measuring their thermal impedance curve, $Z_{\mathrm{th}}(\mathrm{t})$, in combination with their electrical characteristics, the $I_{\mathrm{F}}\left(V_{\mathrm{F}}\right)$ curves, and their radiant flux and luminous flux characteristics, $\Phi_{\mathrm{e}}\left(I_{\mathrm{F}}\right)$ and $\Phi_{\mathrm{e}}\left(I_{\mathrm{F}}\right)$, respectively in compliance with JEDEC's LED thermal testing standards [JEDEC $2012 \mathrm{a}$, b] and CIE's most recent recommendations regarding the optical measurements of high power LEDs [CIE 2017]. Since LEDs in practice are operated in a constant DC forward current operating mode, their electrical characteristics are rather provided as $V_{F}\left(I_{F}\right)$ functions. When the electrical and light output characteristics are measured at a constant junction temperature, $T_{\mathrm{J}}$, they are called iso-thermal IVL characteristics. The junction temperature of the LEDs can be set to a known value TJ by either of the methods recommended by CIE or JEDEC [Bein et al, 2017].

An LED package is completely characterised if the iso-thermal IVL characteristics are obtained for the relevant range of the forward current and junction temperature, thus sets of $V_{F}\left(I_{F}, T_{J}\right)$ and $\Phi_{e}\left(I_{F}, T_{J}\right)$ functions obtained. For the round-robin test performed at the beginning of the Delphi4LED project the minimal recommended set of the LED operating points determined by the forward current and junction temperature points $\left(I_{\mathrm{F}}, T_{\mathrm{J}}\right)$ were defined [Poppe et al 2017]. These recommended operating points for LEDs binned at $I_{\mathrm{F}}=350 \mathrm{~mA}$ and $T_{\mathrm{J}}=85^{\circ} \mathrm{C}$ are summarized in Table 1.

Table 1 - Typical selection of junction temperature and forward current combinations for the measurement of isothermal IVL characteristics of mid-power LEDs [Poppe et al 2017]. Column corresponding to binning temperature and row corresponding to binning current are highlighted in red.

\begin{tabular}{|c|c|c|c|c|c|}
\hline$T_{\text {J }}\left[{ }^{\circ} \mathbf{C}\right]$ & $\mathbf{3 0}$ & $\mathbf{5 0}$ & $\mathbf{7 0}$ & $\mathbf{8 5}$ & $\mathbf{1 1 0}$ \\
\hline \multirow{5}{*}{$I_{F}[\mathrm{~mA}]$} & 20 & 20 & 20 & 20 & - \\
\cline { 2 - 6 } & 30 & 30 & 30 & 30 & - \\
\cline { 2 - 6 } & 60 & 60 & 60 & 60 & - \\
\cline { 2 - 6 } & 100 & 100 & 100 & 100 & - \\
\cline { 2 - 6 } & 350 & 350 & 350 & 350 & 350 \\
\cline { 2 - 6 } & 500 & 500 & 500 & 500 & 500 \\
\cline { 2 - 6 } & - & 700 & 700 & 700 & 700 \\
\cline { 2 - 6 } & - & - & 1000 & 1000 & 1000 \\
\hline
\end{tabular}

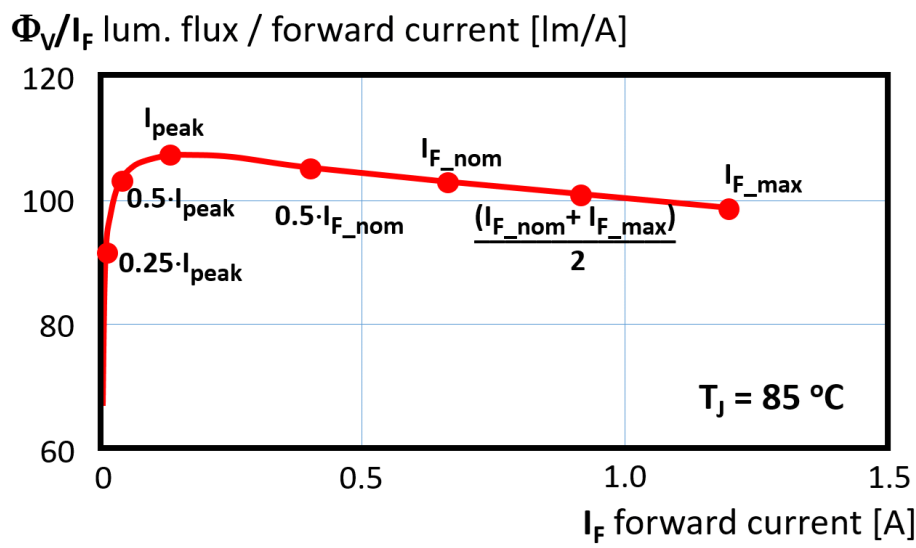

Figure 1 - A typical LED efficacy diagram with forward current values suggested for measurement of isothermal IVL characteristics [Poppe et al 2017].

The basis of this recommendation is that with these forward current values the measured characteristics include LED operating domains both below and above the forward current that 
corresponds to the peak efficacy, at the relevant junction temperature values, see Figure 1. With such sets of operating points, parameters of LEDs' multi-domain models can be well fitted to the model equations. For the measurements, using a combined thermal and radiometric/photometric test setup is recommended that complies with JEDEC's and CIE's standards and recommendations; see e.g. [Farkas and Poppe 2014].

\subsection{A multi-domain LED model}

According to the modular, hierarchical approach of the Delphi4LED project [Marin et al 2019], the multi-domain behaviour of LEDs is captured on chip level, using so called Spice-like models of LED chips [Poppe et al 2019]. The thermal properties of the mechanical package structure is represented by a so called (thermal) boundary condition independent compact thermal model of the LED package [Bornoff 2019]. These two models combined represent the multi-domain behaviour of an LED package.

Parameters of such models, if identified from LED characteristics of individual LED package samples are representative to the samples themselves. In this section we provide an overview of the multi-domain LED model that we used in the study of the sample-to-sample variability. The variability of the thermal model parameters were studied first, and the results have already been widely published [Sari et el 2017], [Bornoff et al 2018], [Mérelle et al 2018], [Bornoff et al 2019].

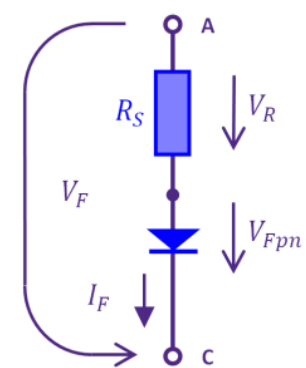

a)

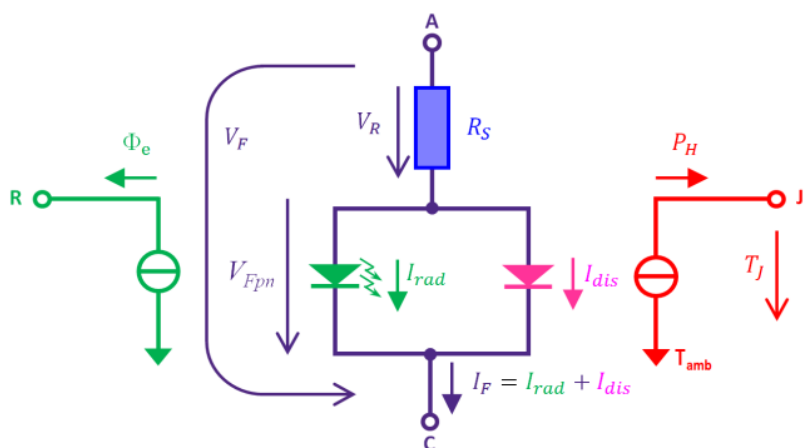

b)

Figure 2 - LED models aimed at circuit simulation with Spice-like circuit simulators: a) and LED considered as a plain, "electrical only diode", b) internal topology of a real multi-domain LED model, that can be implemented in a Spice-like circuit simulator; based on [Poppe at al 2019 ].

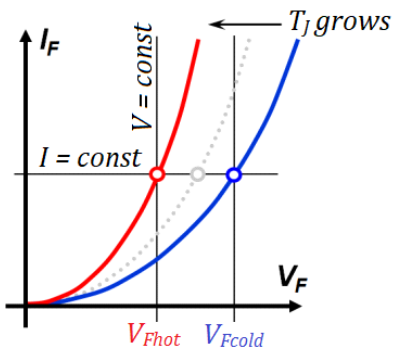

a)

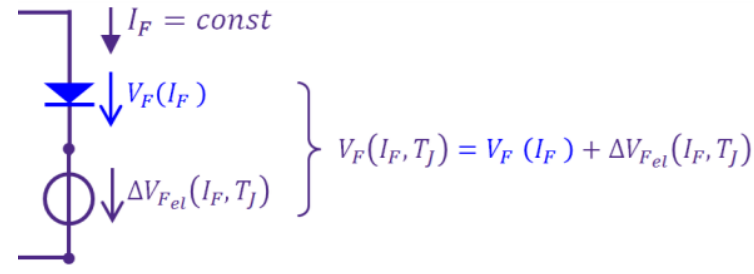

b)

Figure 3 - Temperature dependence of the forward voltage of a diode at constant forward current; b) Modelling the effect of self-heating for both the pn-junction and the series resistance by a single temperature dependent source [Poppe at al 2019].

Figure 2 presents various LED models aimed at simulation to be performed by so called Spice-like electrical circuit simulators. In Figure $2 a$ the topology of the standard, "electrical only" diode model is shown, for which LED vendors often share model parameter sets on their product support websites. The drawback of such traditional models is that there are valid only for a single junction temperature (or for a limited range thereof) and they cannot be used to predict light output properties consistently with the electrical characteristics at any junction temperature value. To overcome this issue, multi-domain Spice-like circuit models have been 
proposed with the basic concept of representing the forward current component associated with the light output, Irad and the heat dissipation, $I_{\text {dis, }}$ as shown in Figure $2 \mathrm{~b}$. The model equations of such a model have been recently reformulated to the typical constant forward DC. Using the notations of Figure 2 and applying Shockley's diode model to the constant forward current driven case, the complete multi-domain operation of an LED chip can be represented by the following set of equations:

$$
\begin{aligned}
& V_{F}=I_{F} \cdot R_{S}+V_{F p n} . \\
& V_{F p n}=m \cdot V_{T} \cdot\left[\ln \left(\frac{I_{F}}{I_{0}}\right)+1\right] \\
& R_{S}\left(T_{J}\right)=R_{S 0}+S_{R S 1} \cdot\left(T_{J}-T_{\text {ref }}\right)+S_{R S 2} \cdot\left(T_{J}-T_{r e f}\right)^{2} \\
& R_{S 0}=R_{S}\left(T_{\text {ref }}\right) .
\end{aligned}
$$

where $T_{\text {ref }}$ is an arbitrary chosen reference value of the junction temperature. For the internal junction voltage it is true that it is the sum of the voltage drops along the elements of the model of the radiative LED branch:

$$
V_{F p n}=V_{F p n_{\text {rad }}}+I_{\text {rad }} \cdot R_{R}=m_{\text {rad }} \cdot V_{T} \cdot \ln \left[\left(\frac{I_{\text {rad }}}{I_{0_{\text {rad }}}}\right)+1\right]+I_{\text {rad }} \cdot R_{R}
$$

In the model of the radiative branch the resistor $R_{\mathrm{R}}$ represents the optical losses that are also

\begin{tabular}{|c|c|c|c|}
\hline \multicolumn{4}{|l|}{ Input quantities: $I_{F}, T_{J}$} \\
\hline \multicolumn{4}{|c|}{ Output quantities: $V_{F}, P_{H}, \Phi_{\mathrm{e}}, \Phi_{\mathrm{V}}$} \\
\hline Model parameters & & & $\begin{array}{l}\text { Symbol in Spice diode } \\
\text { model }\end{array}$ \\
\hline Thermal & \multicolumn{2}{|c|}{$T_{\text {ref }}, V_{T}$} & TNOM, UT \\
\hline Electrical & entire LED & radiative branch only & \\
\hline $\begin{array}{l}\text { diode internal pn- } \\
\text { junctions at } T_{\text {ref }}\end{array}$ & $I_{0}, m$ & $I_{0_{\text {rad }}}, m_{\text {rad }}$ & IS, $\mathrm{N}$ \\
\hline resistors at $T_{\text {ref }}$ & $R_{S}$ & $R_{R}$ & $\begin{array}{l}\mathbf{R S}=0 \text { to switch of } \\
\text { Spice's model of the } \\
\text { series resistance }\end{array}$ \\
\hline $\begin{array}{l}\text { Coefficients of the model } \\
\text { of the " } \Delta V_{F} \text { generators" }\end{array}$ & $\begin{array}{ll}a_{e l}, & b_{e l}, c_{e l}, \\
d_{e l}, & e_{e l}, f_{e l}\end{array}$ & $\begin{array}{c}a_{\text {rad }}, b_{\text {rad }}, c_{\text {rad }}, \\
d_{\text {rad }}, e_{\text {rad }}, f_{\text {rad }}\end{array}$ & - \\
\hline $\begin{array}{l}\text { Coefficients of the efficacy } \\
\text { of radiation model }\end{array}$ & \multicolumn{2}{|c|}{$\begin{array}{c}a_{\text {Kap }}, b_{\text {Kap }}, c_{\text {Kap }}, d_{\text {Kap }} \\
e_{\text {Kap }}, f_{\text {Kap }}, g_{\text {Kap }}, h_{\text {Kap }}, i_{\text {Kap }}\end{array}$} & - \\
\hline
\end{tabular}
converted to heat. The "internal junction voltage" of the radiative branch, $V_{\text {Fradpn, is identified }}$ by solving Eq. (4).

Table 2 - Summary of the set of the parameters of the generic Spice compatible multi-domain LED model [Poppe at al 2019].

Assuming a temperature independent diode model (as in a generic, "electrical only" Spice circuit solver) the junction temperature induced change of the overall forward voltage at constant forward current driven mode of operation (Figure 3a) still can be represented by an extra temperature controlled voltage source in series, as shown in Figure $3 \mathrm{~b}$. The current and temperature dependence of this source can be described by the following empirical formula:

$$
\begin{aligned}
\Delta V_{F e l}= & \left(a_{e l} \cdot I_{F}^{2}+b_{e l} \cdot I_{F}+c_{e l}\right) \cdot\left(T_{J}^{2}-T_{r e f}^{2}\right)+ \\
& \left(d_{e l} \cdot I_{F}^{2}+e_{e l} \cdot I_{F}+f_{e l}\right) \cdot\left(T_{J}-T_{r e f}\right) .
\end{aligned}
$$

In a similar approach, the temperature dependence of the voltage of the radiative branch of the LED model can be described as follows:

$$
\begin{array}{r}
\Delta V_{F_{\text {rad }}}=\left(a_{\text {rad }} \cdot I_{F}^{2}+b_{\text {rad }} \cdot I_{F}+c_{\text {rad }}\right) \cdot\left(T_{J}^{2}-T_{r e f}^{2}\right)+ \\
\left(d_{\text {rad }} \cdot I_{F}^{2}+e_{r a d} \cdot I_{F}+f_{r a d}\right) \cdot\left(T_{J}-T_{r e f}\right) .
\end{array}
$$


This way the emitted total radiant flux is calculated as follows:

$$
\Phi_{\mathrm{e}}=\left(V_{F p n_{\text {rad }}}+\Delta V_{F_{\text {rad }}}\right) \cdot I_{\text {rad }} .
$$

From this the heat dissipation and the emitted to total luminous flux are calculated as follows:

$$
P_{H}=I_{F} \cdot V_{F}-\Phi_{\mathrm{e}},
$$

and

$$
\Phi_{\mathrm{V}}\left(I_{F}, T_{J}\right)=\Phi_{\mathrm{e}}\left(I_{F}, T_{J}\right) \cdot \mathrm{K}\left(I_{F}, T_{J}\right) .
$$

where $\mathrm{K}$ is the efficacy of radiation which can be well modelled for LEDs by a bi-quadratic formula:

$$
\begin{aligned}
\mathrm{K}\left(I_{F}, T_{J}\right)= & \left(a_{\text {Kap }} \cdot T_{J}^{2}+b_{\text {Kap }} \cdot T_{J}+c_{\text {Kap }}\right) \cdot I_{F}^{2}+\left(d_{\text {Kap }} \cdot T_{J}^{2}+e_{\text {Kap }} \cdot T_{J}+f_{K a p}\right) \cdot I_{F}+ \\
& \left(g_{\text {Kap }} \cdot T_{J}^{2}+h_{\text {Kap }} \cdot T_{J}+i_{\text {Kap }}\right) .
\end{aligned}
$$

In Table 2 we provide the summary of the parameters of the multi-domain LED chip model represented by Eqs. (1)-(10).

The question that we investigate in a subsequent section of this paper is how these model parameters are affected by the sample-to-sample variations and what conclusions can be drawn from the statistical analysis of the empirically obtained distributions of these model parameters.

\section{Variability analysis of LED package characteristics}

For the analysis of the sample-to-sample variations of LED package model parameters a separate set of LEDs have been used (from one of the leading LED vendors): 11 samples of royal blue and 11 samples of phosphor converted white LEDs have been measured and modelled. We had the assumption that the white LEDs ware based on the same blue pump chips as used in the royal blue samples. All samples were chosen and supplied from the same, narrow bin.

Both $Z_{\text {th }}(t)$ curves and corresponding thermal structure functions and the representative set of the isothermal IVL characteristics have been measured.at the operating points listed in Table 1. From the obtained thermal structure functions a compact thermal model of the package + MCPCB assembly structure for every LED has been created and analysed.

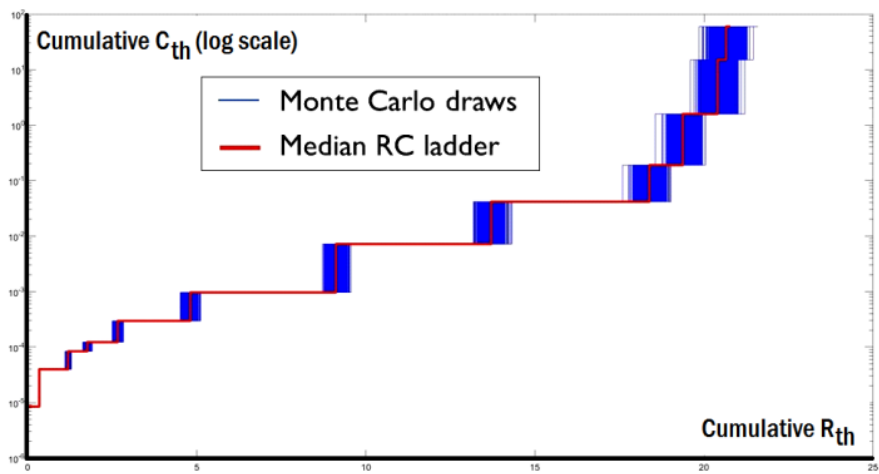

a)

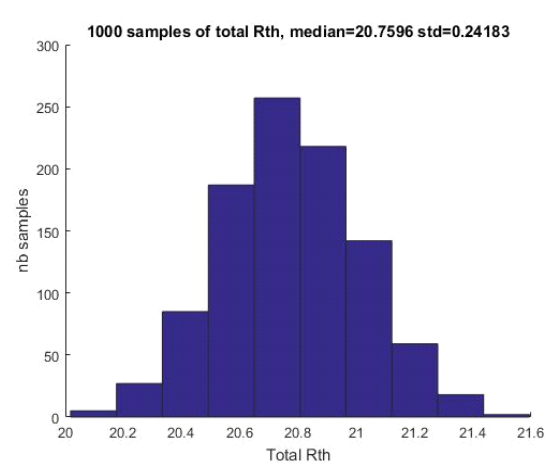

b)

Figure 4 - Step-wise approximation of the structure function of the "median" sample of a population of 11 royal blue LEDs with Monte Carlo modelled scatter of the individual partial thermal resistances of the heat-flow path; b) the histogram of the simulated value distribution of one of the stages RC ladder model of the structure function [Merelle et al 2018]. 


\subsection{Variability of the thermal properties of the junction to ambient heat flow path}

In this analysis first a median sample (for both the royal blue and the white LED package populations) was chosen. We chose the sample the total junction-to-ambient thermal reistance, $R_{\text {thJA }}$ of which was closest to the average value of the property. For the sake of simplicity the investigated thermal model was a one dimensional RC Cauer ladder model, the element values of which were obtained by step wise approximation of the structure function of the heat-flow path. A viable assumption was to fix the thermal capacitance values at the ones obtained for the median device. After this the variations of the values of the partial thermal resistances of the heat-flow path depicted in the model were investigated and modelled [Bornoff et al 2018], [Mérelle et al 2018], [Bornoff et al 2019]. These values properly represent the variation of the so called local thermal resistance along the heat-flow path.

\subsection{A first look at the variability of the multi-domain characteristics}

In Figure 5 we present the measured isothermal electrical characteristics of the 11 white LED samples measured. As it can be seen, the sets of characteristics corresponding to the different junction temperatures overlap. In order to obtain a better idea of the possible behaviour of the measured LEDs we a set of normalized characteristics have been generated. Such are the relative efficacy plots such as presented in Figure 1.

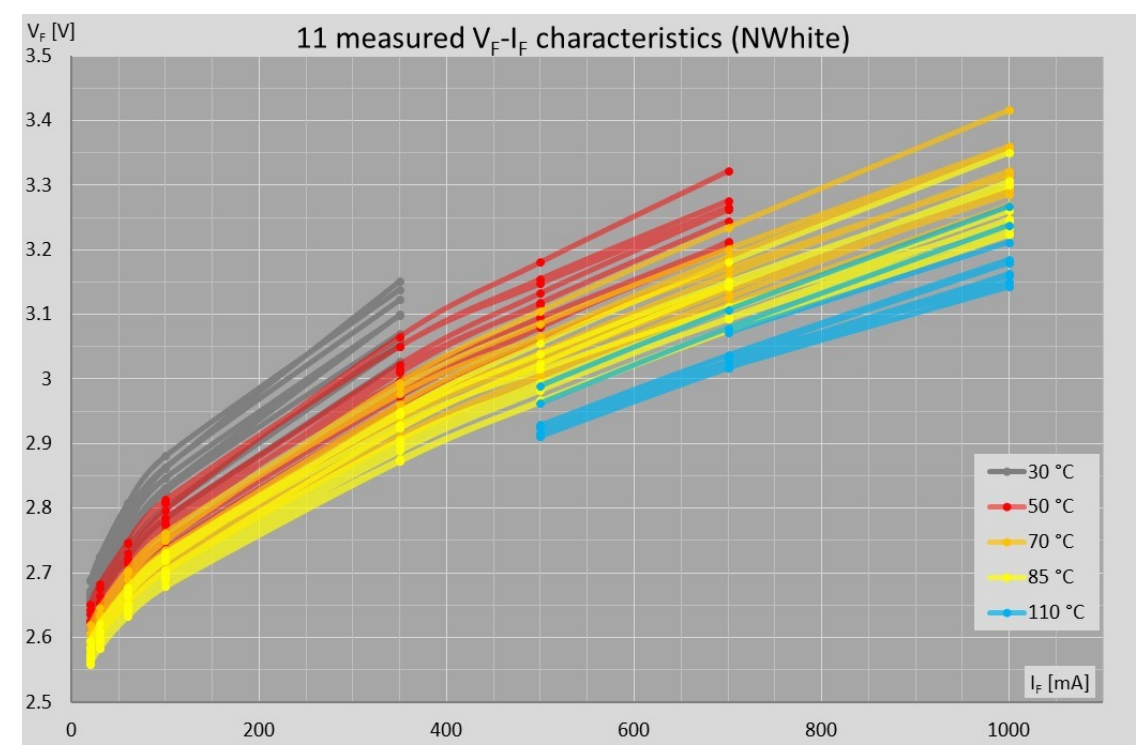

Figure 5 - Isothermal forward voltage - forward current characteristics of 11 phosphor converted neutral white LED samples, measured at different junction temperatures between 30 ${ }^{\circ} \mathrm{C}$ and $110^{\circ} \mathrm{C}$.

Besides the efficacy diagrams, plots representing the temperature dependence of the relative luminous fluxes of the samples were also generated. Using such plots we could have a better idea how the LED samples behaved. Typical results obtained for the phosphor converted white and royal blue samples out of the investigated populations are shown in Figure 6 and in Figure 7 , respectively.

As seen in these diagrams, there are plots that might correspond to two different subpopulations, both in case of the white and the royal blue LED samples, however, all the plots represent equal values for the binning current and temperature as they intersect there.

It is important to note, that the flux characteristics such as shown in Figure 1 and presented in Figure $6 \mathrm{a}$ and in Figure $7 \mathrm{~b}$ are fingerprints of a given LED package (chip + encapsulation with phosphor, if applicable) and may reveal information about the possible sub-population of LEDs present in a given population from even a narrow binning class. Therefore, reporting electrical and optical properties related to the binning current and binning temperature only may not be sufficient for end-users, especially when they aim to model their LED application precisely. It is also important to mention, that this limited test data reporting (restricted to 
providing LED properties to the binning current and binning temperature, e.g. to $I_{\mathrm{F}}=350 \mathrm{~mA}$ and $T \mathrm{~J}=85^{\circ} \mathrm{C}$ only) can hide LED vendors' standard practice of mixing LED chips from various sources and selling them under the same LED type and binning class.

\subsection{First results of the statistical analysis of the multi-domain model parameters}

Though, from a statistical point of view the number of samples was too low (11 - 11 samples in both LED categories discussed) an attempt to make a correct statistical analysis of the multi-domain LED behaviour was performed, through the analysis of the extracted multidomain LED model parameters (see Table 2 and Eqs. (1)-(10)). The questions addressed were:

- how to define a "median device" from the point of view of multi-domain LED operation represented by the isothermal IVL characteristics of the individual samples,

- how distributions of the model parameters summarised in Table 2 can be represented by statistical methods (correlation analysis among the parameters, analysis of distributions of the parameters),

- and how the identified statistical features can be modelled in a Monte Carlo scheme in order to allow to simulate how the sample-to-sample variations can be represented in a system level model, such as complete luminaire compact model [Poppe 2017] into which instances of the chip level multi-domain LED model are included.

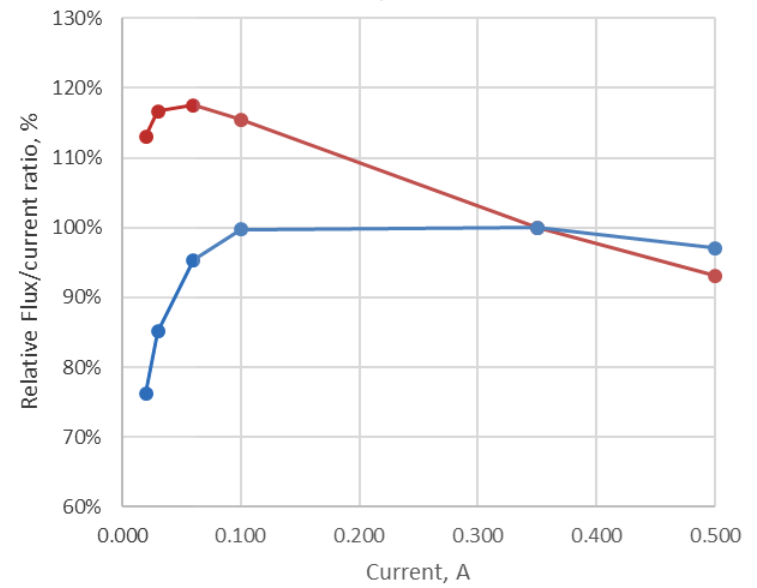

a)

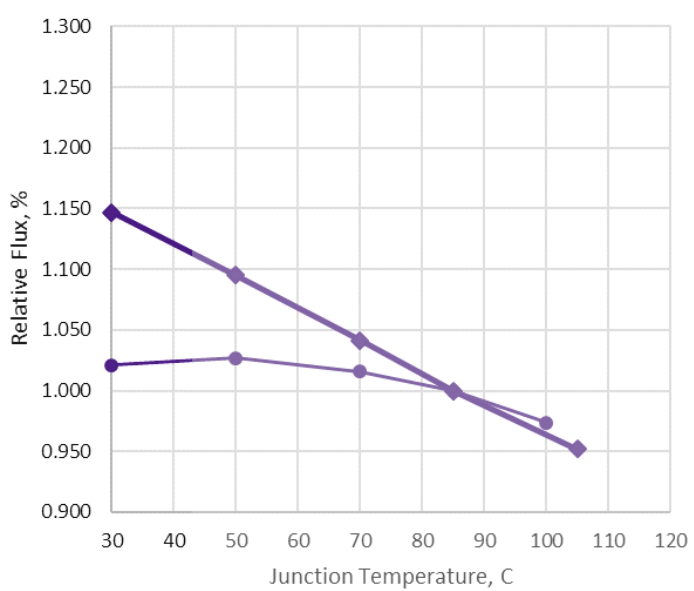

b)

Figure 6 - Test results of two samples of a given white power LED type from the same bin: a) relative efficacy diagrams measured at $T \mathrm{~J}=85^{\circ} \mathrm{C}, \mathrm{b}$ ) temperature dependence of the relative luminous flux measured at $I_{\mathrm{F}}=\mathbf{3 5 0} \mathrm{mA}$.

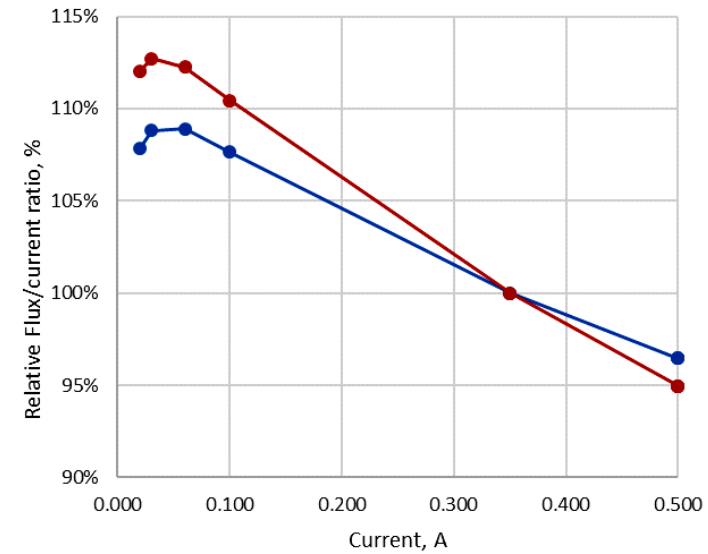

a)

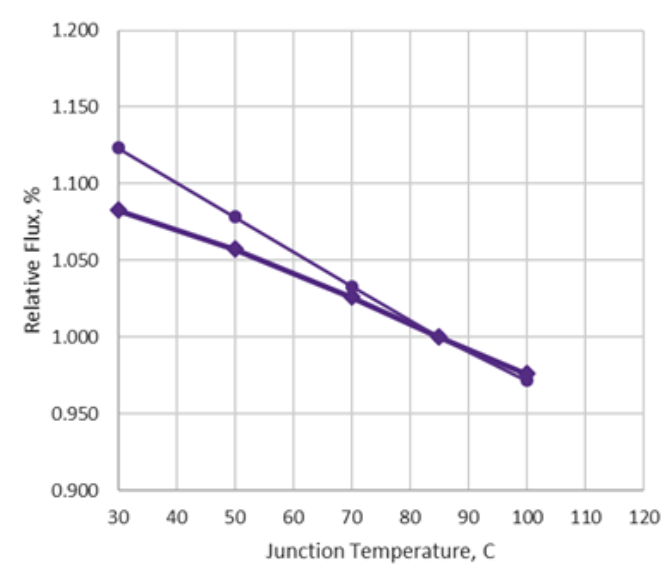

b)

Figure 7 - Test results of two samples of a given blue power LED type from the same bin: a) relative efficacy diagrams measured at $T_{J}=85^{\circ} \mathrm{C}, \mathrm{b}$ ) temperature dependence of the relative luminous flux measured at $I_{\mathrm{F}}=350 \mathrm{~mA}$. The nominal peak wavelength of the blue LEDs corresponds to the blue peak of the spectra of the white LEDs shown in Figure 6. 
The statistical analysis revealed a few "outlier" samples that were discared from some of the farther analysis. In Figure 8 some results of the correlation analysis of the royal blue samples are shown by means of depicting their correlation matrices. As seen in Figure 8, parameters $I_{0}$ and $m$ are strongly correlated. This statement also applies to the white LED sample population. Parameter $R_{\mathrm{s}}$ was strongly anti-correlated with this parameters for the royal blue samples (shown in the figure) but not for the white LEDs. Parameters /orad and $m_{\text {rad }}$ again were strongly correlated, both for the blue and white LEDs, $R_{R}$ was also correlated with them in case of the blue LEDs, but not for the white ones.

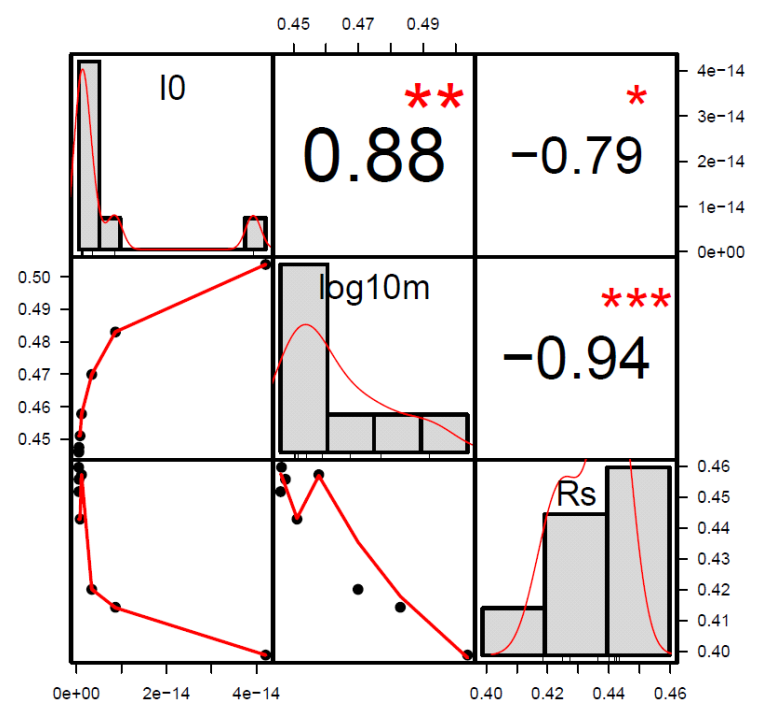

a)

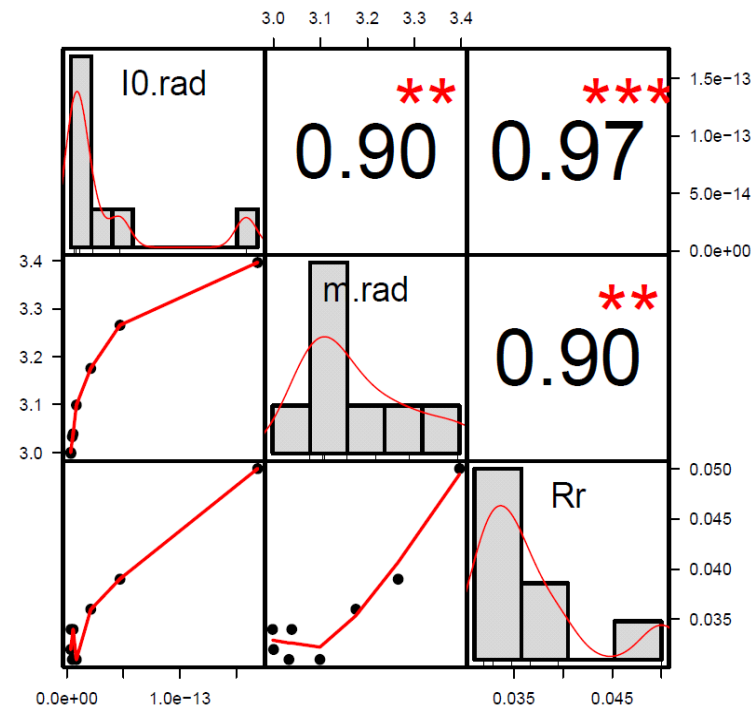

b)

Figure 8 - Correlation matrices of the Shockley model parameters of a) $/ 0, m$ and $R s$ of the "main diode" used in Eqs.(1), (2) and b) lorad, $m_{\text {rad }}$ and $R_{R}$ of the "radiative diode", represented by Eqs. (2), (4). (Outlier samples were discarded in this analysis.)

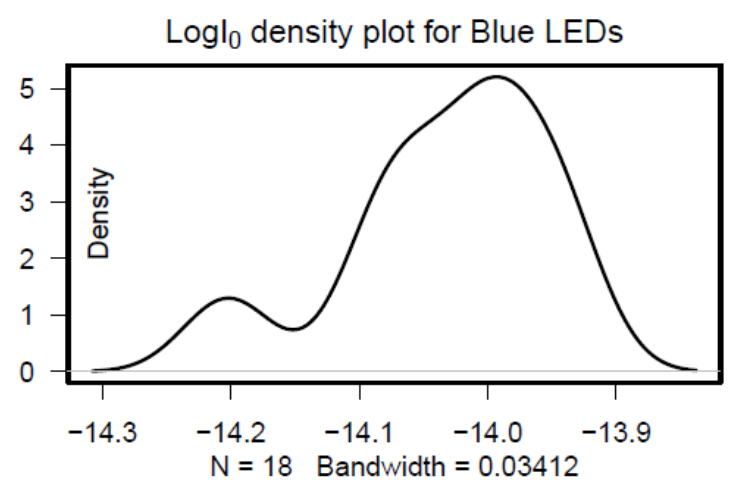

a)

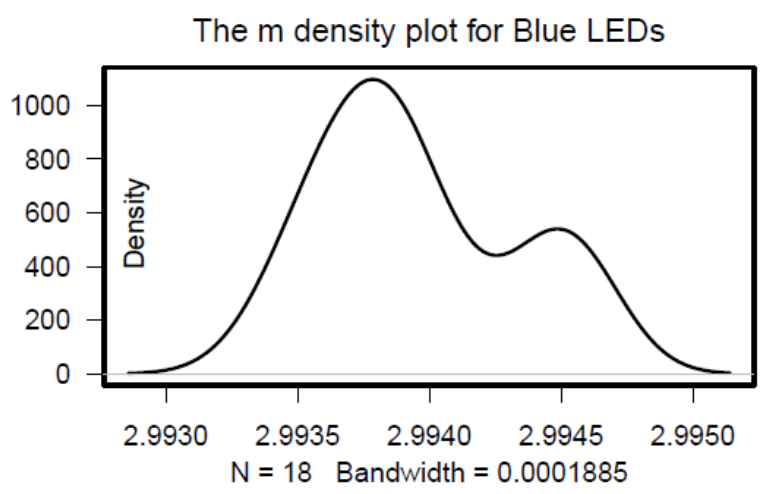

b)

Figure 9 - Density plots of the parameter distributions of the royal blue LED samples: a) the $I_{0}$ parameters, b) the $m$ parameters.

In case of the parameters of the " $\Delta V_{F}$ " generators used to represent temperature dependence in an electrical-only circuit simulation environment a rather complex correlation - anticorrelation pattern was found for both LED populations. The same applies for the $\mathrm{K}\left(I_{F}, T_{J}\right)$ model given by Eq. (10).

In a Monte Carlo model set up later, for groups of correlated parameters a single draw was made for the main parameter of a given group. In case of losely correlated parameters independent draws were implemented.

In a second analysis the parameter distributions were analysed by means of density functions (normal and lognormal plots were created). Examples for such plots for the royal blue LEDs is 
given in Figure 9 for parameters $I_{0}$ and $m$. Similar plots were also generated for the $R \mathrm{~s}$ parameters.

Both normal and lognormal density plots of the parameters $10, \mathrm{~m}$ and $\mathrm{RS}$ of the royal blue LEDs give a strong indication of the possibility of 2 sub-populations present in the investigated LED population (well distinguishable peaks in the density functions).This corresponds to the findings based on the efficacy diagrams shown in Figure 7. Regarding the parameters of the "radiative diode" the results if this analysis is less conclusive, perhaps due to the statistically low number of samples analysed.

\subsection{Selection of a "median device"}

In terms of the multi-domain behaviour selection of a median device representative to the characterised LED population is not straightforward. One could prefer to nominate an existing physical device that is the closest to the "average" multi-domain behaviour of the population, or one may define a "virtual" LED device, representing this average. Our team investigated the following options:

1. nominate one of the physical samples based on the binning criteria of the LED vendor,

2. derive the $\eta_{\mathrm{e}}\left(I_{F}, T_{J}\right)$ efficiency, $\eta_{\mathrm{V}}\left(I_{F}, T_{J}\right)$ efficacy and $\mathrm{K}\left(I_{F}, T_{J}\right)$ luminous efficacy of radiation surfaces and nominate the devise who's such surfaces in average are closest to the average surfaces of the population (in our approach a combined ranking of the Euclidan distances were used),

3. create a virtual device for which the value of each multi-domain model parameter is chosen as the most likely one from the distribution of the given parameter,

4. create a virtual device that is characterized by a set of isothermal IVL curves obtained as averages of the measured curves.

From this list options 2 and 4 are the easiest to implement. An example for the implementation of method 2 from the above list is shown in Figure 10.

\begin{tabular}{|l|r|r|r|}
\hline Sample & R.Dev $\eta$ e & R.Dev $\eta v$ & R.Dev K \\
\hline $1 \mathrm{~A}$ & $5.67 \%$ & $3.44 \%$ & $2.22 \%$ \\
\hline $1 \mathrm{~B}$ & $2.94 \%$ & $3.09 \%$ & $0.63 \%$ \\
\hline $1 \mathrm{C}$ & $5.69 \%$ & $5.16 \%$ & $3.90 \%$ \\
\hline $1 \mathrm{D}$ & $2.99 \%$ & $2.79 \%$ & $1.03 \%$ \\
\hline $1 \mathrm{E}$ & $2.14 \%$ & $1.78 \%$ & $0.47 \%$ \\
\hline $1 \mathrm{~F}$ & $2.72 \%$ & $2.98 \%$ & $0.61 \%$ \\
\hline $2 \mathrm{~A}$ & $5.14 \%$ & $4.83 \%$ & $0.97 \%$ \\
\hline $2 \mathrm{~B}$ & $9.06 \%$ & $8.92 \%$ & $0.85 \%$ \\
\hline $2 \mathrm{C}$ & $3.78 \%$ & $6.98 \%$ & $0.18 \%$ \\
\hline $2 \mathrm{D}$ & $1.65 \%$ & $1.93 \%$ & $0.72 \%$ \\
\hline $2 \mathrm{E}$ & $6.39 \%$ & $6.53 \%$ & $0.53 \%$ \\
\hline
\end{tabular}

\begin{tabular}{l|r|r|r|r|} 
Sample & R.Dev $\eta$ e & R.Dev $\eta v$ & R.Dev K & sum_rank \\
\hline $1 \mathrm{E}$ & 2 & 1 & 2 & 5 \\
\hline $2 \mathrm{D}$ & 1 & 2 & 6 & 9 \\
\hline $1 \mathrm{~F}$ & 3 & 4 & 4 & 11 \\
\hline $1 \mathrm{~B}$ & 4 & 5 & 5 & 14 \\
\hline $1 \mathrm{D}$ & 5 & 3 & 9 & 17 \\
\hline $2 \mathrm{C}$ & 6 & 10 & 1 & 17 \\
\hline $2 \mathrm{~A}$ & 7 & 7 & 8 & 22 \\
\hline $2 \mathrm{E}$ & 10 & 9 & 3 & 22 \\
\hline $1 \mathrm{~A}$ & 8 & 6 & 10 & 24 \\
\hline $1 \mathrm{C}$ & 9 & 8 & 11 & 28 \\
\hline $2 B$ & 11 & 11 & 7 & 29 \\
\hline
\end{tabular}

Combined iso IVL Closest to ave

a)

b)

Figure 10 - Nominating a physical LED sample as "median device" based on the combined ranking according to the relative deviations from the different efficacy/efficiency surfaces.

\section{Conclusions}

In this paper we presented how multi-domain characterization of LEDs (by means of measurement and modelling of their isothermal IVL characteristics) can help reveal hidden details of an LED population chosen from the same, even very narrow binning class. Both relative efficacy curves and a deeper statistical analysis of multi-domain model parameters obtained for two LED populations of 11-11 royal blue samples of a mainstream LED type of a leading vendor suggest that even though all samples were from the same binning class (with perfectly matching properties at the binning current and temperature), from the wider perspective of the multi-domain behaviour might represent multiple LED types. 


\section{Acknowledgement}

This research received funding from the European Union's Horizon 2020 research and innovation program through the H2020 ECSEL project Delphi4LED (grant agreement 692465). Co-financing of the Delphi4LED project by the national public research funding organisations of the participating countries is also acknowledged.

\section{References}

Bein et al 2017. BEIN, M. C. HEGEDÜS, J. HANTOS, G. GAÁL, L. FARKAS, G. RENCZ, M.; POPPE, A. Comparison of two alternative junction temperature setting methods aimed for thermal and optical testing of high power LEDs. In: Proceedings of the 23rd International Workshop on THERMal INvestigation of ICs and Systems (THERMINIC'17), Amsterdam, The Netherlands, 27-29 September 2017, DOI: 10.1109/THERMINIC.2017.8233838

Bornoff et al 2018. BORNOFF, R. MÉRELLE, T.. SARI, J. . DI BUCCHIANICO, A. FARKAS, G. Quantified Insights into LED Variability, In: Proceedings of the 24th International Workshop on THERMal INvestigation of ICs and Systems (THERMINIC'18), Stockholm, Sweden, 26-28 September 2018, DOI: 10.1109/THERMINIC.2018.8593315

Bornoff 2019. BORNOFF, R. Extraction of Boundary Condition Independent Dynamic Compact Thermal Models of LEDs - a Delphi4LED Methodology. Energies, 2019, Special Issue on Thermal and Electro-thermal System Simulation

Bornoff et al 2019. BORNOFF, R.; MÉRELLE, T.; SARI, J.; DI BUCCHIANICO, A.; FARKAS, G. A Methodology to Determine the Sites of Variability in an LED Assembly. In: Proceedings of the 35th Semiconductor Thermal Measurement and Management Symposium (SEMI-THERM'19), San Jose, USA, 18-22 March 2019, pp. 1-6.

CIE 2017. CIE Technical Report 225:2017. Optical Measurement of High-Power LEDs. ISBN 978-3-902842-12-1, 2017, DOI: 10.25039/TR.225.2017

Farkas and Poppe 2014. FARKAS, G. POPPE, A. Thermal testing of LEDs. , In: Thermal Management for LED Applications, Lasance, C. J. M., Poppe , A., Eds; Springer, 2014, pp. 73-165. DOI: 10.1007/978-1-4614-5091-7_4

JEDEC 2012a. JEDEC JESD51-51 Standard "Implementation of the Electrical Test Method for the Measurement of Real Thermal Resistance and Impedance of Light-Emitting Diodes with Exposed Cooling" (2012)

JEDEC 2012b. JEDEC JESD51-52 Standard "Guidelines for Combining CIE 127:2007 Total Flux Measurements with Thermal Measurements of LEDs with Exposed Cooling Surface" (2012)

Martin et al 2019. MARTIN, G. MARTY, C. BORNOFF, R. POPPE, A. ONUSHKIN, G. RENCZ, M. YU, J. Luminaire Digital Design Flow with Multi-Domain Digital Twins of LEDs. Energies, 2019, Special Issue on Thermal and Electro-thermal System Simulation

Marty et al 2018. MARTY, C. YU, J. MARTIN, G. BORNOFF, R. POPPE, A. FOURNIER, D. MORARD, E. Design flow for the development of optimized LED luminaires using multidomain compact model simulations. In: Proceedings of the 24th International Workshop on THERMal INvestigation of ICs and Systems (THERMINIC'18) Stockholm, Sweden, 2628 September 2018, DOI: 10.1109/THERMINIC.2018.8593318

Mérelle et al 2018. MÉRELLE, T. BORNOFF, R. ONUSHKIN, G. GAÁL, L. FARKAS, G. POPPE, A. HANTOS, G. SARI, J. DI BUCCHIANICO, A. Modeling and quantifying LED variability, In: Proceedings of the 2018 LED Professional Symposium (LpS2018), Bregenz, Austria, 25-27 September 2018, (ISBN: 978-3-9503209-9-2), pp. 194-206

Poppe 2017. POPPE, A. Simulation of LED Based Luminaires by Using Multi-Domain Compact Models of LEDs and Compact Thermal Models of their Thermal Environment. Microelectronics Reliability, 2017 Vol. 72(5), pp. 65-74.,

DOI: $10.1016 /$ j.microrel.2017.03.039 
Poppe et al 2017. POPPE, A. FARKAS, G. SZABÓ, F. JOLY, J. THOMÉ, J. YU, J. BOSSCHAARTL, K. JUNTUNEN, E.; VAUMORIN, E. DI BUCCHIANICO, A. MÉRELLE, T. Inter Laboratory Comparison of LED Measurements Aimed as Input for Multi-Domain Compact Model Development within a European-wide R\&D Project. In: Proceedings of the Conference on "Smarter Lighting for Better Life" at the CIE Midterm Meeting, Jeju, SK, pp. 569-579, DOI: 10.25039/x44.2017.PP16

Poppe et al 2019. POPPE, A. FARKAS, G. GAÁL, L. HANTOS, G. HEGEDÜS, J. RENCZ, M. Multi-domain modelling of LEDs for supporting virtual prototyping of luminaires", Energies, 2019, Special Issue on Thermal and Electro-thermal System Simulation

Sari et al 2017. SARI, J. MÉRELLE, T. DI BUCCHIANICO, A. BRETON, D. Delphi4LED: LED Measurements and Variability Analysis. In: Proceedings of the 23rd International Workshop on THERMal INvestigation of ICs and Systems (THERMINIC'17), Amsterdam, The Netherlands, 27-29 September 2017, DOI: 10.1109/THERMINIC.2017.8233797 